\title{
Multimode Heavy Robots Motion Control and Powertrain Optimization
}

\author{
Denis Pogosov \\ Electronics and Mechatronics Department, Southern Federal University, Rostov-on-Don (Russia)
}

The paper presents synthesis of a control system of a heavy industrial mobile robot with optimization of its energy consumption. The introduced methods acts to fuel saving. We arranged a special motion control mode of the engine works with a constant power, so the optimal engine mode is achieved and no braking needed during the robot motion. The paper presents a method for optimization the rotor flux of electric powertrain induction motors. The introduced solutions are universal about power characteristics of traction motors and to their working mode. This allows the system to control the traction motors with non-hyperbolic characteristics as well as above rated speed without changing the structure of the control system.

Motion control is based on algorithmic framework of the Position-trajectory control. We enlarged the Positiontrajectory method to a class of systems which motion coupled with the constant engine power, as well as to a class of systems, which have redundant structure. We introduced the multimode control method able to soft switch the motion modes.

\section{Keywords: Mobile robot, Nonlinear control, Constant power motion, Position-trajectory control, Fuel save, Powertrain optimisation, Multimode control}

\section{INTRODUCTION}

Designing a robotic control system of heavy mobile robots (HMR) for industrial and civil engineering with energy optimization is a comprehensive multi-tasking problem. This complexity follows from the structure of the controlled object, which has several elements, that energy optimization is a separate analysis and carried out by different methods, in general.

A typical structure of a tractive drive of HMR includes [1] a diesel internal combustion engine (DE), electric powertrain system (EP) and wheeled movers.

Overcoming of rough terrain obstacles, during the movement, requires increasing $\mathrm{DE}$ power that is a negative impact on the fuel consumption. From the internal combustion engines theory [2-3] is well known that high dynamics of the engine impair its quality characteristics to reduce the emissions, especially NOx. Reduction NOx emissions most efficiently at a low combustion temperature, which is most easily achieved with constant engine power and constant crankshaft speed. There should be noted that the optimal area of $\mathrm{DE}$ is in a neighbourhood of the nominal load point. Just in this optimal area the minimum of fuel consumption is achieved at significantly low engine dynamics. As evidenced by the foregoing causes the task of arrangement a HMR movement with constant power and constant DE crankshaft speed, mainly in the nominal point.

Motion with high accuracy on the desired trajectory with constant power of the main engine requires high powertrain dynamics, which leads to the relevant tasks to improve EP efficiency and reduce losses.

\section{MATHEMATICAL MODEL OF MOBILE ROBOT EQUIPPED AC ELECTRIC TRANSMISSION}

\subsection{Chassis Model}

Modern heavy mobile robots have different constructions. Mostly they are presented as with a twoaxle vehicle with driving rear and steering front axles. At this time actively developed systems with steering-drive axles, for example Belaz 7571 with two and prospective ETF Truck with five steering-drive axles. In this paper we used an universal generalized vector-matrix model of HMR for the design of the motion control systems of wide class HMR, as well as a simplified model of classic HMR chassis for synthesis a control system example and its simulation.

The generalised model in vector-matrix form given as follows [4]:

$$
\begin{gathered}
\dot{X}=M^{-1}\left(L_{2} \tilde{Q}-F_{r}-F_{N}\right) \\
\dot{Y}=\Sigma(P, \gamma)
\end{gathered}
$$

where: $Y-$ is vector of the chassis position $P$ and orientation (yaw) $\gamma, \Sigma()-$. is a nonlinear function of coordinates transformation, $L_{2}$ - is matrix of torque-force transformation, $X-$ is vector internal coordinates (of steering angle and driven wheels speed), $\tilde{Q}-$ is vector of internal coordinates derivatives (torque), $F_{r}$ - is vector of a motion resistance, $M$ - is matrix of mass and inertia parameters, $F_{N}$-is vector of immeasurable disturbances.

This generalized model we can complete describe the wheeled robots and tracked robots. No needed to linearization the model or dynamic decomposition.

The simplified model of classic HMR chassis is particular case of system (1)-(2) and contains these two equations. We represent the specializing model in vectormatrix form as follows [5-6]:

$$
\begin{gathered}
X=\left[\begin{array}{ll}
\theta & \omega
\end{array}\right]^{T} \\
Y=\left[\begin{array}{ll}
P & \gamma
\end{array}\right]^{T} \\
\dot{P}=r k_{w g} \omega\left[\begin{array}{l}
\cos \theta \cos \gamma-a^{-1} \sin \theta \sin \gamma \\
\cos \theta \cos \gamma+a^{-1} \sin \theta \sin \gamma
\end{array}\right] \\
\dot{\gamma}=a^{-1} r k_{w g} \omega \sin \theta \\
k_{s} \ddot{\theta}=U_{s t}-h b^{-1} \sin \beta
\end{gathered}
$$




$$
\begin{gathered}
M=\operatorname{diag}(1, m r) \\
L_{2}=\operatorname{diag}\left(-r^{-1} k_{w g}^{-1}, 1\right) \\
F_{r}=f_{g} m g\left(\cos \alpha\left[\begin{array}{c}
0 \\
\cos \beta-\frac{h}{a} \tan \alpha
\end{array}\right]+\left[\begin{array}{c}
0 \\
\sin \alpha
\end{array}\right]\right)+F_{a}
\end{gathered}
$$

where: $\theta$ - is steering angle, $\omega-$ is driven shaft speed (before wheel gear), $r$ - is the wheel radius, $k_{w g}-$ is the wheel gear ratio, $a$ - is distance between axles, $b$ - is distance between wheels, $h$ - is center of gravity height, $m$ - is HMR mass, $F_{a}$ - is the air resistance, $g$ - is free fall acceleration constant, $k_{s}-$ is inertial steering constant, $U_{s t}-$ is control variable of the steering unit, $\alpha$ - longitudinal (like pitch) angle, $\beta$ - is lateral (like roll) angle, $f_{g}$ - is the ground coefficient, $\operatorname{diag}($.$) - is operator$ to create a diagonal matrix from the operator arguments. No immeasurable disturbances this case.

\subsection{Electric Transmission Model}

Frequently modern electric powertrains of HMR are based on AC induction motors (IM) as a traction drive, AC synchronous generators (SG), as well as inverters with DC link.

High quality control of AC drives is possible by the vector control methods [7-10]. According to the method, all electric units are represent as vectors in the twodimensional reference frame $d, q$.

\subsubsection{Model of Induction Motor}

We represent the model of IM in the $d, q$ frame, that rotating synchronously with the rotor flux, according to the FOC [7-13] method. The model in scalar form given as follows [7-13]:

$$
\begin{gathered}
U_{d}=\sigma L_{s} \dot{I}_{d}-\sigma L_{s} \omega_{\Phi} I_{q}+R_{1} I_{d}-L_{r}^{-2} L_{m} R_{r} \Phi \\
U_{q}=\sigma L_{s} \dot{I}_{q}+\sigma L_{s} \omega_{\Phi} I_{d}+R_{s} I_{q}+L_{r}^{-1} L_{m} \omega_{\Phi} \Phi \\
R_{1}=R_{s}+L_{r}^{-2} L_{m}^{2} R_{r} \\
L_{r} R_{r}^{-1} \dot{\Phi}=L_{m} I_{d}-\Phi \\
Q=1.5 Z_{p} L_{m} L_{r}^{-1} I_{q} \Phi-k_{f} \omega \\
\omega_{\Phi}-Z_{p} \omega=\frac{L_{m} R_{r} I_{q}}{L_{r} \Phi}
\end{gathered}
$$

where: $U_{d}, U_{q}$ - are stator voltage components in the reference frame, $I_{d}, I_{q}$ - are stator currents components in the reference frame, $R_{s}$ - is the stator resistance, $R_{r}-$ is the rotor resistance, $L_{s}-$ is the stator inductance, $L_{r}-$ is the rotor inductance, $L_{m}-$ is the mutual inductance, $\sigma$ - is the motor leakage inductance, $\Phi$ - is the rotor flux, $Q$ - is the motor torque, $Z_{p}$ - is pole pairs quantity, $\omega_{\Phi}$ - is the reference frame speed, $k_{f}$ - is a friction coefficient.

The $L_{s}, L_{m}, L_{r}$ and $\sigma$ are approximated functions depends on main magnetic flux $\Phi_{m}$, which estimates by measured voltage and current of the stator and the rotor speed. This is way to take into account the saturation of magnetic system if IM.

\subsubsection{Model of Inverter}

Hardware implementation of the inverter can be quite different [7-10], so we restricted only by most important relationships.

We assume the inverter as two-stage voltage converter. The first stage is DC link, based on diode rectifiers with capacitance filter. The second stage is selfexcited voltage inverter, based on six semiconductor switches.

The inverter realises necessary voltage to IM by vector PWM method. Figure 1 shown the diagram of six base vectors U1-U6, from that realises the necessary vector $U$.

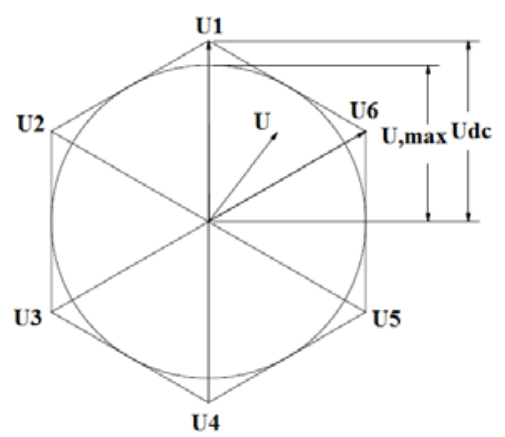

Figure 1: Vector PWM diagram

So, maximal voltage calculated as follows:

$$
U_{\max }=\frac{\sqrt{3}}{2} U_{d c}
$$

where: $U_{\max }$ - is IM maximal voltage unrestricted by the hexagonal area, $U_{d c}-$ is voltage on DC link.

Software implementation of it can be carried out by variety of known methods [7-8, 10].

Second matter relationship is power loses of the invertor, they are sum of constant and switching loses:

$$
P_{\text {loss }, \text { inv }}=I^{2}\left(R_{s c}+P_{s w} f_{P W M}\right)
$$

where: $P_{\text {loss,inv }}$ - is inverter losses, $I$ - is current through inverter, $R_{s c}$ - is semiconductor switch resistance, $P_{s w}-$ is elementary switching resistance correspond to losses (depends on switching time), $f_{P W M}-$ is PWM base frequency.

At the current angles divisible by 30 degrees, the right summand in (18) is equal to zero.

\subsubsection{Model of Synchronous Generator}

We represent the model of SG in the $d, q$ frame, that rotating synchronously with the rotor. The model in scalar form given as follows [7-13]:

$$
\begin{gathered}
U_{g d}=L_{g d} \hat{\dot{I}}_{g d}+L_{g m} \dot{I}_{f}+R_{g} I_{g d}+\omega_{g} L_{g q} I_{g q} \\
U_{g q}=L_{g q} \hat{\dot{I}}_{g q}-\omega_{g} L_{g d} I_{g d}+R_{g} I_{g q}-\omega_{g} L_{g m} I_{f} \\
U_{f}=R_{f} I_{f}+L_{f} \dot{I}_{f}+L_{g m} \hat{\dot{I}}_{g d} \\
Q_{\text {load }}=1.5 Z_{g p} I_{g q}\left(L_{g m} I_{f}+L_{\Delta} I_{g d}\right)+k_{f g} \omega_{g}
\end{gathered}
$$

where: $U_{g d}, U_{g q}$ - are stator voltage components in the reference frame, $I_{g d}, I_{g q}$ - are stator currents components in the reference frame, $\hat{\dot{I}}_{g d}, \hat{\dot{I}}_{g q}$ - are estimated stator currents components derivatives in the reference frame, 
$R_{g}$ - is the stator resistance, $R_{f}-$ is the inductor resistance, $L_{g d}, L_{g q}$ - are stator inductance components, $L_{\Delta}$ - is the stator inductance difference, $L_{g m}-$ is the mutual inductance, $L_{f}$ - is the inductor inductance, $U_{f}-$ is inductor voltage, $Q_{\text {load }}-$ is the load torque, $Z_{g p}-$ is pole pairs quantity, $\omega_{g}-$ is the reference frame speed, $k_{f g}$ - is a friction coefficient.

This model describes generalized SG with a salient poles rotor, mostly used as EP generators. In particular case of round rotor $L_{\Delta}$ is equal to zero. $L_{f}$ is a nonlinear function of inductor current. This is way to take into account the saturation of magnetic system of SG.

\subsubsection{Coordinates Transformation}

Due to the fact that the above models of IM and SG obtained for the two-dimensional reference frame and most real AC electric machines have three phases (some synchronous generators can be six-phase), there is necessary to coordinates transformation from the natural phase system to $d, q$ and vice versa. $8,10,14]$ :

The coordinates transformation given as follows [7-

$$
\begin{gathered}
R(.)=\left[\begin{array}{cc}
\cos (.) & \sin (.) \\
-\sin (.) & \cos (.)
\end{array}\right] \\
U_{3 I M}=P_{S} R^{-1}\left(\gamma_{\omega}\right)\left[\begin{array}{ll}
U_{d} & U_{q}
\end{array}\right]^{T} \\
U_{3 S G}=P_{S} R^{-1}\left(\gamma_{R}\right)\left[\begin{array}{ll}
U_{g d} & U_{g q}
\end{array}\right]^{T} \\
P_{S}=\left[\begin{array}{ccc}
1 & -1 / 2 & -1 / 2 \\
0 & \sqrt{3} / 2 & -\sqrt{3} / 2
\end{array}\right]^{T}
\end{gathered}
$$

where: $U_{3 I M}-$ is a vector of IM phase voltages, $U_{3 S G}-$ is a vector of SG phase voltages, $\gamma_{\omega}-$ is the angle of IM rotor flux, $\gamma_{R}-$ is the angle of SD rotor, $R()-$. is a rotation matrix.

Quite similar transformation may be obtained for currents or other variables of the electric machines.

\subsection{Diesel Engine Model}

A generalised model of DE torque balance in scalar form given as follows [2]:

$$
J_{e} \dot{\omega}_{e}=M_{\text {ind }}-M_{f r}-M_{\text {pump }}
$$

where: $J_{e}-$ is the engine inertia, $M_{i n d}-$ is the indicated engine torque, $M_{f r}-$ is the friction engine torque, $M_{\text {pump }}$ - is the pump torque.

Engine torque components can be represented as follows [2, 14-15]:

$$
\begin{gathered}
M_{\text {ind }}=\frac{k_{F} U_{F} Q_{L H V} R_{g a s} T_{i m}\left(e_{0}+e_{1} \omega_{e}+e_{2} \omega_{e}^{2}\right)}{\eta_{V} V_{d} P_{i m}} \\
\eta_{V}=e_{3}+e_{4} \omega_{e}+e_{5} \omega_{e}^{2} \\
M_{f r}=\frac{1000 V_{d}\left(e_{6}+e_{7} \omega_{e}+e_{8} \omega_{e}^{2}\right)}{2 \pi n_{R}} \\
M_{\text {pump }}=e_{9} P_{i m}+e_{10}
\end{gathered}
$$

where: $U_{F}-$ is a fuel rate, $Q_{L H V}-$ is the fuel lower heating value; $R_{\text {gas }}-$ is the gas constant, $P_{i m}, T_{i m}-$ are pressure and temperature in intake manifold, respectively, $V_{d}$ - is the engine volume, $\eta_{V}$-is volumetric efficiency, $n_{R}$ - is number of revolutions for each power stroke per cycle, $k_{F}, e_{0}-e_{10}-$ are constants. We assume measurable of intake manifold pressure and temperature.

\section{OPTIMISATION AND CONSIDERATION OF ENERGY LOSSES}

We assume two types of energy losses; the first is optimizable losses, the second is not. The efficiency of EP is a nonlinear function of the consumed power. So, to stabilize the power of DE, the control system should use remain energy, exclude all losses.

\subsection{IM Energy Losses and Optimisation}

Energy optimization techniques of AC motors are concentrated [16-19] on solutions based on the Lagrange approach. In [17, 20] the Lagrange method is used both with the Kuhn-Tucker theorem, that extends the method to cases of constraints set by the inequalities. It is used in [16-17] for synthesis a control of a motor on the boundary modes of operation. Presented in [21] solutions are designed to optimize both components of the stator current or contain extreme conditions where IM is operating at the maximum voltage or current.

Mathematical model of IM with squirrel-cage rotor is represented by (11)-(16). Thus, with respect to (11), (12) and (15) the orthogonal components of the current vector represent IM as a two dimensional object. As follows from (15), $I_{q}$ component of stator current allows to control IM torque and $I_{d}$ component allows to flux rotor control, i.e. electromagnetic state of IM. In the case of a constant rotor flux, control of $I_{q}$ channel allows nearly infinitely fast control of IM torque (15), thereby ensuring a high quickaction performance of a motion control system. However, retention of the rotor flux at a constant (nominal) level is a negative impact on energy consumption. Since the rotor time constant $L_{r} R_{r}^{-1}$ has a large value versus $\sigma L_{s}$, forced control of $\Phi$ will attend energy loss.

For motion control systems is necessary to guarantee a smooth transition from a system with maximum performance properties to a system with maximum energy efficiency properties, predominantly in the intermediate area of operation. This can be achieved by separating the control channels of IM torque and the rotor flux, with a possibility of separate quality of transients on each channel. One matter note there is, this separating is a formal approach, like separating the stator current to two components. So, there is no decomposition of the physical model. For example, in applications where the quickaction is more important than energy efficiency, increasing the transient time of the rotor flux changing, will allow the control system to fast torque control. Tuning the rotor flux to optimal value at long duration thereby will be without energy losses of force of this process. That is, without deterioration of performance characteristics by capture energy from $I_{q}$ component of stator current during the torque transient. 
This necessitates the optimization of some function, which is energy loss function of IM, depending on $I_{d}$ component of stator current (or the rotor flux) and independent of $I_{q}$ component. The function may be obtained from the equation of power balance equation, by subtracting therefrom the net power (mechanical work), as well as non-optimizable power losses. We consider total loses and optimizable loses function $\Lambda$ in steady-state as the following equation [16-18, 20, 22]:

$$
\begin{gathered}
P_{\text {loss }, I M}=\frac{4}{3} \frac{d W}{d t}+\frac{2}{3} P_{\text {copper }}+\frac{2}{3} P_{\text {core }}+P_{f r} \\
\Lambda=\frac{4}{3} W+\frac{2}{3} P_{\text {copper }}+\frac{2}{3} P_{\text {core }} \\
W=\sigma L_{s} I_{s}^{2}+L_{r}^{-1} \Phi^{2} \\
P_{\text {copper }}=\left(R_{s}+R_{r} \frac{L_{m}^{2}}{L_{r}^{2}}\right) I_{s}^{2}-\frac{R_{r}}{L_{r}^{2}} \Phi^{2} \\
P_{\text {core }}=\frac{L_{m}^{2}}{L_{r}^{2}}\left(k_{h}\left|\omega_{\Phi}\right|+k_{e} \omega_{\Phi}^{2}\right) \Phi^{2} \\
I_{s}^{2}=\frac{\Phi^{2}}{L_{m}^{2}}+\frac{4 L_{r}^{2} Q^{2}}{9 Z_{p}^{2} L_{m}^{2} \Phi^{2}}
\end{gathered}
$$

where: $P_{\text {loss }, I M}$ - is total IM losses, $P_{\text {copper }}$ - is copper losses of the rotor and the stator, $P_{\text {core }}-$ is stator iron losses (at a low sliding core losses of the rotor usually neglected [17]), $P_{f r}-$ is friction and windage losses, $W-$ is the stored energy in the magnetic system of IM, $k_{e}-$ is eddy currents constant, $k_{h}$-is hysteresis constant, $I_{s}-$ is the Euclidean norm of stator current vector.

The magnetic energy $W$ is the first integral of the magnetic power. Minimizing this is needed to reduce reactive currents.

Using equation (37) we able to represent the function (33) as a function of the rotor flux.

We compute the partial derivative of the function (33) of the rotor flux and given follows equations:

$$
\begin{gathered}
\frac{\partial \Lambda}{\partial \Phi}=\frac{4}{3} \frac{\partial W}{\partial \Phi}+\frac{2}{3} \frac{\partial P_{\text {copper }}}{\partial \Phi}+\frac{2}{3} \frac{\partial P_{\text {core }}}{\partial \Phi}=0 \\
\frac{\partial I_{s}^{2}}{\partial \Phi}=\frac{2 \Phi}{L_{m}^{2}}-\frac{8 L_{r}^{2} Q^{2}}{9 Z_{p}^{2} L_{m}^{2} \Phi^{3}} \\
\frac{\partial W}{\partial \Phi}=\sigma L_{s} \frac{\partial I_{s}^{2}}{\partial \Phi}+2 L_{r}^{-1} \Phi \\
\frac{\partial P_{\text {copper }}}{\partial \Phi}=\left(R_{s}+R_{r} \frac{L_{m}^{2}}{L_{r}^{2}}\right) \frac{\partial I_{s}^{2}}{\partial \Phi}-\frac{R_{r} \Phi}{L_{r}^{2}} \\
\frac{\partial P_{\text {core }}}{\partial \Phi}=\frac{2 L_{m}^{2}}{L_{r}^{2}}\left(k_{h}\left|\omega_{\Phi}\right|+k_{e} \omega_{\Phi}^{2}\right) \Phi
\end{gathered}
$$
equation:

Then, we equal (38) to zero and solve the resulting

$$
\Phi_{\text {opt }}=\sqrt{Q L_{\Phi}\left(\omega_{\Phi}, \Phi_{m}\right)}
$$

where: $\Phi_{\text {opt }}-$ is the optimal rotor flux, $L_{\Phi}\left(\omega_{\Phi}, \Phi_{m}\right)-$ is the nonliner function, aproximated from solving (38).

\subsection{SG Energy Losses and Optimisation}

We will introduce the SG losses by follows vectormatrix equations:

$$
\begin{gathered}
P_{\text {loss }, S G}=\frac{3}{2}\left(I_{g}^{T} L_{g} \hat{\dot{I}}_{g}+I_{g}^{T}\left(R_{G}+\omega_{g} L_{g} D\right) I_{g}\right)+k_{f g} \omega_{g} \\
L_{g}=\operatorname{diag}\left(L_{g d}, L_{g q}\right) \\
P_{\text {loss }, f}=R_{f} I_{f}^{2}+L_{f} \dot{I}_{f} I_{f}
\end{gathered}
$$

where: $P_{\text {loss }, S G}-$ is total SG losses, $I_{g}$ - is vector of stator current, $\hat{\dot{I}}_{g}$ - is estimated stator current derivative, $R_{G}$ - is the stator resistance diagonal matrix, $P_{\text {loss }, f}-$ is the inductor losses, $L_{g}-$ is stator inductance matrix, $L_{g m}$ - is the mutual inductance, $D$ - is skew-symmetric matrix.

The windage losses should depend on square-law of a shaft speed, but in this paper they (15), (22), (32), (44) are described as linear functions of correspond electrical machine shaft speed. The reason is, that SG and IM has high power capacity and they usually have units for active cooling. So they have not large impellers for cooling and the windage losses can be approximated by linear functions.

There should be noted about SG optimisation. The components of SG voltage and current in the reference frame are depends on its Euclidean norms and angle between the vector and $q$ axle. The Euclidean norms are fully determined from necessary IM voltage and currents (11), (12) and (17). The angle between $I_{g}$ and $U_{g}$ is depend on power factor and skew angle $\varphi$, namely:

$$
\varphi=\frac{I_{d} U_{d}+I_{q} U_{q}}{\|I\|\|U\|}-\arctan \left(\omega_{\Phi} C_{d c}\right)
$$

where: $\|I\|,\|U\|$ - are Euclidean norms of IM voltage and current, $C_{d c}$ - is capacitance of DC link filter.

The angle between $q$ axes and $U_{g}$ is depend on load angle $\hat{\theta}_{g}$, which estimated from the current components, after measure the phases current, we assume. Hence SG is fully determined object, and there is no redundancy, which might have optimised.

\subsection{Total losses}

The total losses we assume are losses of SG, IM, and the inverter. There we separate the total losses to two types. The first is static losses, which is time independent. The second is dynamic losses, which is time depends.

The static losses equation is get from (17), (18), (32), (35), (36), (44) and (46) and given as follows:

$$
\begin{gathered}
P_{\text {loss }, \text { static }}=I_{s}^{T} \tilde{R} I_{s}+L_{3} \Phi^{2}+k_{f} \omega+k_{f g} \omega_{g}+R_{f} I_{f}^{2} \\
\tilde{R}=\frac{3}{\sqrt{3}}\left(R_{G}+\omega_{g} L_{g} D\right)+R_{2} \\
R_{2}=\frac{1}{2} \operatorname{diag}\left(1.5 R_{1}+R_{s c}+P_{s w} f_{P W M}\right) \\
L_{3}=\frac{3}{2}\left(\frac{L_{m}^{2}\left(k_{h}\left|\omega_{\Phi}\right|+k_{e} \omega_{\Phi}^{2}\right)-R_{r}}{L_{r}^{2}}\right)
\end{gathered}
$$

The dynamic losses equation is get from (17), (32), (34), (35), (44) and (46) and given as follows:

$$
P_{\text {loss, dynamic }}=I_{s}^{T} \tilde{L} \dot{I}_{s}+2 L_{r}^{-1} \Phi \dot{\Phi}+L_{f} I_{f} \dot{I}_{f}
$$




$$
\tilde{L}=\frac{3}{\sqrt{3}} L_{g}+\frac{3}{2} L_{S}
$$

where: $L_{S}$ - is stator inductance diagonal matrix.

For control synthesis we will use both losses equations (48) and (52).

\section{MOTION CONTROL ALGORITHM SYNTHESYS}

The control object represented by (1)-(17), (19)(22), (28)-(31) is essentially nonlinear and multilinked object. There should be noted an impossibility of usage of any linear control system or a systems which working with a decomposed original model of a control object. This is due to the fact, that such systems adequately control an essentially nonlinear objects at significantly narrow area of operation. Thus, for the synthesis of control algorithms, we need the basis control methods of nonlinear and multilinked objects. As such method we choose the Position-trajectory control method [4, 23-26]. This method enables to control the moving objects along on a desired path (trajectory goal) and at a constant speed. Another feature of the method is equality of number of control goals to controlled variables. These present a problem for redundant systems control.

Therefore we supplement the Position-trajectory control method with a motion control with constant power (not just speed) and we supplemented the method with an additional governor of redundant variable (as a sub-goal) in terms of the Position-trajectory control.

\subsection{Control goal and control problem definition}

We define the control system goal for the synthesis as movement on a trajectory at a desired constant power of traction motor (IM).

We set a trajectory goal of HMR by following vector-matrix equation [4], as a function $N_{1}$ of external coordinates (4) in implicit form [27]:

$$
\Psi_{T}=\left[\begin{array}{ll}
N_{1}(P) & 0_{2 \times 1}
\end{array}\right]^{T}
$$

We set power consumption at desired level $w j$ at steady state, by the following vector equation, as a function of internal coordinates and their derivatives (2)(3):

$$
\begin{gathered}
\tilde{W}=\left[\begin{array}{lll}
0 & N_{2} & N_{3}
\end{array}\right]^{T} \\
N_{2}=-|X|^{T} M \dot{X}+|X|^{T} F_{R}+P_{\text {loss,static }}-w j \\
N_{3}=\Phi^{2}-\Phi_{\text {opt }}^{2}
\end{gathered}
$$

We assume few outputs of control system. The output group is traction side controls, namely steering control (7) and IM voltage (11)-(12), which also used as the inverter control input:

$$
U=\left[\begin{array}{ll}
U_{d} & U_{q}
\end{array}\right]^{T}
$$

The second output group is generation side controls, voltage of SG inductor (21) and fuel rate of DE (28).
We define the desired character of TR motion as a closed-loop system by follows system of vector-matrix equations:

$$
\begin{gathered}
\Psi=\Psi_{T}+A \dot{\Psi}_{T} \\
\widetilde{\Psi}=\Psi+T \tilde{W}+T \dot{\Psi} \\
\widetilde{\widetilde{\Psi}}=\widetilde{\Psi}+C \dot{\widetilde{\Psi}}
\end{gathered}
$$

where: $A, T$ and $C$ - are diagonal matrixes of constant coefficients, which define the desired character of the system action and transient [4, 23-26].

First of all, we present some assumptions, which are taking place in synthesis of the control system.

According to the well-known Lenz principle of the constancy an interlinkage, the resulting magnetic flux cannot change abruptly. Therefore, the rotor flux changes slow, so $\ddot{\Phi} \cong 0$, and then:

$$
\begin{gathered}
\dot{\Phi} \equiv L_{m} \dot{I}_{d}-J_{L m} \hat{\dot{\Phi}}_{m} I_{d} \\
J_{L m}=\frac{\partial L_{m}}{\partial \Phi_{m}}
\end{gathered}
$$

For some minor computations, can be assumed that:

$$
\frac{d}{d t}\left(\frac{L_{m}^{2}}{L_{r}^{2}}\right) \cong 0
$$

Because SG shaft and DE shaft are connected directly $\omega_{g}=\omega_{e}$.

According to the Position-trajectory control method [4, 23-26], we differentiate equations (1)-(2) simultaneously with (3)-(10):

$$
\begin{gathered}
\ddot{Y}=J_{\Sigma X} \dot{X}+J_{\Sigma Y} \dot{Y} \\
\dddot{Y}=J_{\Sigma X} \ddot{X}+\Gamma_{\Sigma X} \dot{X}+J_{\Sigma Y} \ddot{Y}+\Gamma_{\Sigma Y} \dot{Y} \\
\ddot{X}=M^{-1}\left(L_{2} \dot{\tilde{Q}}-\dot{F}_{r}-\dot{F}_{N}\right) \\
J_{\Sigma X}=\frac{\partial \Sigma}{\partial X^{T}}, J_{\Sigma Y}=\frac{\partial \Sigma}{\partial Y^{T}}, \Gamma_{a}=\frac{d J_{a}}{d t}, a=\Sigma X, \Sigma Y
\end{gathered}
$$

Therefore we differentiate (15) and simultaneously with (62) get the following vector-matrix equation:

$$
\begin{gathered}
\dot{\tilde{Q}}=K_{Q 5}\left[\begin{array}{c}
U_{s t} \\
\dot{I}
\end{array}\right]+K_{Q 6} \tilde{Q}+K_{Q 7} I \\
K_{Q 5}=\left[\begin{array}{cc}
k_{s}^{-1} & 0_{1 \times 2} \\
0 & K_{m}\left[\begin{array}{cc}
L_{m} I_{q} & \Phi
\end{array}\right]
\end{array}\right], K_{m}=\frac{3 Z_{p} L_{m}}{2 L_{r}} \\
K_{Q 6}=\left[\begin{array}{cc}
0 & 0 \\
\frac{J_{L m} \hat{\dot{\Phi}}_{m}\left(L_{r}-1\right)}{L_{m} L_{r}} & 0 \\
0 & 0
\end{array}\right] \\
K_{Q 7}=\left[\begin{array}{cc}
0 & 0 \\
K_{m} J_{L m} \hat{\dot{\Phi}}_{m} & 0
\end{array}\right]
\end{gathered}
$$

Then, we differentiate (54)-(55) according to (59)(61) and with substitution of (65)-(67), (69) into the result, and then solving the result equation relative the vector of steering controls and current directive from (69), we obtain follows vector-matrix equation:

$$
\begin{gathered}
{\left[\begin{array}{c}
U_{s t} \\
\dot{I}
\end{array}\right]=-K_{0}^{-1}\left(K_{1} \dot{Y}-K_{3} F_{r}-K_{00} \dot{F}_{r}+K_{4} \tilde{Q}+\Psi_{T}+T \tilde{W}+C T K_{W}+C T K_{6}+K_{8} I+C T K_{9}\right)} \\
K_{00}=C T\left(A J J_{\Sigma X}+J_{W}\right) M^{-1} \\
K_{0}=\left(K_{00}-K_{Z 2}\right) K_{Q 5}+C T K_{P 11} \\
K_{1}=C T A J J_{\Sigma Y}+(T A+C A+C T) J+2 C T A \Gamma
\end{gathered}
$$




$$
\begin{aligned}
& K_{2}=(A+T+C) J+(T A+C A+C T) \Gamma+C T A \dot{\Gamma}+\Gamma_{\Sigma Y}+K_{1} J_{\Sigma Y} \\
& K_{3}=\left(C T K_{P 3}+C T A J \Gamma_{\Sigma X}+K_{1} J_{\Sigma Y}\right) M^{-1} \\
& K_{4}=\left(K_{00}-K_{Z 2}\right) K_{Q 6}-C T K_{Z 3}+K_{3} \\
& K_{8}=\left(K_{00}-K_{Z 2}\right)\left[-k_{s}^{-1} b^{-1} h \sin \beta \quad 0\right]^{T} \\
& K_{9}=\left(K_{00}-K_{Z 2}\right)
\end{aligned}
$$

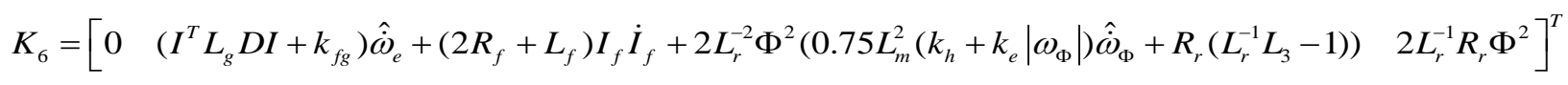

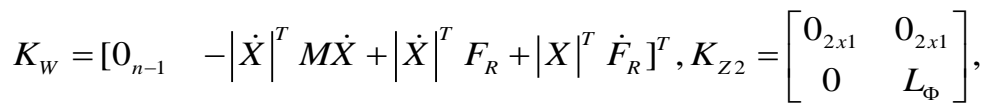

$$
\begin{aligned}
& J_{W}=\left[\begin{array}{ll}
0_{(n-1) x 2} & -|X|^{T} M
\end{array}\right]^{T}, J=\frac{\partial \Psi_{T}}{\partial Y^{T}}, \Gamma=\frac{d J}{d t} \\
& K_{Z 3}=\left[\begin{array}{cc}
0_{2 \times 1} & 0_{2 \times 1} \\
0 & J_{L \Phi} \hat{\dot{\Phi}}_{m}
\end{array}\right], K_{P 3}=\left[\begin{array}{cc}
0 & 0 \\
0 & k_{f} \\
0 & 0
\end{array}\right], K_{P 11}=\left[\begin{array}{cc}
0 & 0_{1 \times 2} \\
0 & I^{T}(\tilde{R}+\tilde{L}) \\
0 & 0_{1 \times 2}
\end{array}\right], K_{51}=\frac{2}{L_{r}}\left[\begin{array}{cc}
0 & 0 \\
\left(L_{3}+1\right) \Phi R_{r} & 0 \\
L_{m} \Phi R_{r} & 0
\end{array}\right]
\end{aligned}
$$

Matrix $K_{6}$ is depends on derivative of the static power loss with addition of dynamic power loss and with assumption (64). The terms $\hat{\dot{\omega}}_{e}, \dot{I}_{f}, \hat{\dot{\omega}}_{\Phi}$ are estimates of correspond variables and represent their current status before computation as the control terms. In other words, for a discrete system they are previous step data.

The system (73) together with (74)-(85) provides us the steering control and current derivative. The voltage on IM is obtained from:

$$
\begin{aligned}
& U=L_{I M} \dot{I}+\left(\omega_{\Phi} L_{I M} D+R_{I M}\right) I+K_{I M} \\
& L_{I M}=\operatorname{diag}\left(\sigma L_{s}\right), R_{I M}=\operatorname{diag}\left(R_{1}, R_{s}\right) \\
& K_{I M}=\operatorname{diag}\left(-L_{r}^{-2} L_{m} R_{r} \Phi, L_{r}^{-1} L_{m} \omega_{\Phi} \Phi\right)
\end{aligned}
$$

As we described before, voltage (86) is going to the inverter input and be used for follows computation of SG inductor voltage:

$$
\begin{gathered}
U_{f}=\left[\begin{array}{ll}
L_{f} & R_{f}
\end{array}\right] K_{f}+\left[\begin{array}{ll}
L_{g m} & 0
\end{array}\right] \hat{\dot{I}}_{g} \\
K_{f}=K_{G 3}^{-1}\left(U_{g}-L_{g} \hat{\dot{I}}_{g}-R_{G} I_{g}-\omega_{e} L_{g} D I_{g}\right) \\
\mathrm{U}_{\mathrm{g}}=\frac{2}{\sqrt{3}} U^{T} U \bar{R}\left(\hat{\theta}_{g}\right) \\
\mathrm{I}_{\mathrm{g}}=I^{T} I \bar{R}\left(\hat{\theta}_{g}+\varphi\right) \\
K_{G 3}=\left[\begin{array}{cc}
L_{g m} & 0 \\
0 & -\omega_{g} L_{g m}
\end{array}\right] \\
\bar{R}(.)=\left[\begin{array}{ll}
\sin (.) & \cos (.)]^{T}
\end{array}\right.
\end{gathered}
$$

Above equations also compute SG voltage (91) and current (92). DE fuel rate is obtained from:

$$
\begin{gathered}
U_{F}=K_{I C E}\left(M_{f r}+M_{\text {pump }}+J_{e} \dot{\omega}_{e}\right) \\
K_{I C E}=\frac{\eta_{V} V_{d} P_{i m}}{k_{F} Q_{L H V} R_{g a s} T_{i m}\left(e_{0}+e_{1} \omega_{e}+e_{2} \omega_{e}^{2}\right)} \\
\dot{\omega}_{e}=\frac{3 Z_{g}}{2 J_{g}} I_{g}^{T}\left(K_{G 1} K_{f}+K_{G 2} I_{g}\right) \\
K_{G 1}=\left[\begin{array}{cc}
0 & 0 \\
0 & L_{g m}
\end{array}\right], K_{G 2}=\left[\begin{array}{cc}
L_{\Delta} & 0 \\
0 & 0
\end{array}\right]
\end{gathered}
$$

The voltages are known, the currents are measured, SG inductor voltage and current no needed to measure. All estimated units getting the value from numerical computations.
So, the control system is fully determined, and basic control outputs are getting from (73), (86), (89) and (95).

\section{MULTIMODE CONTROL}

During HMR operation, it need switching the motion modes (54) and constant speed, or other, obviously.

In case of constant speed mode with desired speed level $v j$, there is new control which put into (73) instead of (55), the new control as follows:

$$
A \tilde{V}=\left[\begin{array}{lll}
0 & X^{T} L^{T} L X-v j^{2} & 0
\end{array}\right]^{T}
$$

All entries in the second row matrices derivatives from the expression (55) equal to zero. In addition, in (74) the $J_{W}$ matrix is need to be changed to follows:

$$
J_{V}=\left[\begin{array}{ccc}
0 & 0 & 0 \\
X^{T} L^{T} L & 0 \\
0 & 0 & 0
\end{array}\right]
$$
equation:

Moreover, need to sum in (78) and (79) the follows

$$
\begin{gathered}
\left(T A\left(C \Gamma_{V}+J_{V}\right)\right) M^{-1} \\
\Gamma_{V}=\frac{d J_{V}}{d t}
\end{gathered}
$$

For example, the modes (54) and (99) needs to be switched, when HMR speed fall under some minimum acceptable speed on a long and high hill, that requiring more power. In this case the control system necessary to switch the motion mode from (54) to (99), and after the hill is traversed, return the motion mode from (99) to (54). In case the current speed or power is different at a certain critical value a new required power or speed, the system will have dynamic shock when switching the mode. To ensure soft switching in this paper we propose a follows procedure:

1) All system controllable matrices (54), (55), (99) and their derivative matrices we increase size to $4 \times 3$.

2) We define a linear function $s_{\text {new }}=k_{n} s_{0}$ of the transition to the new motion mode in the entry of matrixes $A, T, C$, and as well as the function $s_{\text {old }}=-k_{o} s_{0}$ of the elements of the same matrixes, of the old mode. The constants $k_{o}, k_{n}$ should determine same speed of both modes switching time. 
3) So, and the system switches the new mode and $s_{\text {old }}=0$.

4) We need to apply new coefficients to control equations, namely in (73):

$$
\begin{gathered}
T \tilde{W} \rightarrow(T+C \dot{T}) \tilde{W} \\
A \tilde{V} \rightarrow(A+T \dot{A}+C T \dot{A}+C \dot{T} \dot{A}+C \dot{A}) \tilde{V}
\end{gathered}
$$

Then add follows equation into (77):

$$
(T \dot{A}+C \dot{T} \dot{A}+C \dot{T}+C \dot{A}) J+C T \dot{A} \Gamma
$$

Then add follows equation into (76):

$$
(2 C T \dot{A}+C \dot{T A}) J
$$

Then add follows equation into (101):

$$
(C \dot{T} A+C T \dot{A}) J_{V}
$$

The matrixes $\dot{A}, \dot{T}, \dot{C}$ are diagonal matrixes of entries $k_{n},-k_{o}$, corrsponds to the new and old modes, other entries are zero.

If constant operation the matrixes $\dot{A}, \dot{T}, \dot{C}$ are equal to zero and ) and matrices (54), (55), (99) have size $3 \times 3$.

During operation of HMR, some cases of perturbations occurs the control system loss of controllability of the robot.

In order to protect HMR is necessary to employ the control algorithms limitaions. Proposed multimode control able to organize fast and sstable witching bretween regular motion mode and emergency modes as well ass boundary modes of HMR operation.

There should be noted that the development of emergency control systems is a problem, which is an independent research and beyond the scope of this paper.

\section{SIMULATION}

Simulation of introduced control algorithms (73)(98) was accomplished in the software package MATLAB.

Because the control object is highly nonlinear, we assume some simplification for better readable of the simulation results.

Chassis HMR is presented by (1)-(10) and has the following parameters: weight 8 tons, length $8.0 \mathrm{~m}$, width $2.8 \mathrm{~m}$, inertia is $950 \mathrm{~kg} \cdot \mathrm{m}^{2}$, radius of the driven wheel is $0.5 \mathrm{~m}$, secondary gear ratio is $1: 10$. IM is represented by (11)-(16) and have the following parameters: maximum voltage is $1000 \mathrm{~V}$, maximum power is $95 \mathrm{~kW}$, $L_{S}=L_{r}=0.2755, \quad L_{m}=0.2676, \quad \sigma=0.05683$, $R_{r}=0.5625, R_{s}=0.2755$ and $Z_{p}=1$. IM will work on the linear area of its magnetisation characteristic, so all inductances are constant. We neglected the core losses and friction losses. This case the nonlinear function in (43) is a constant and $L_{\Phi}=0.4076$.

SG is represent by (19)-(22) with follows parameters: $L_{g}=0.25, R_{g}=0.497, L_{\Delta}=0, Z_{p}=1$, $L_{f}=0.8$ and $R_{f}=2.95$. We neglected the derivatives of current in (19), (20) and SG inductor loses, so the generator is represented as an inertial part. The inverter losses are neglected too.

DE characterization [14] is shown on Fig.2.

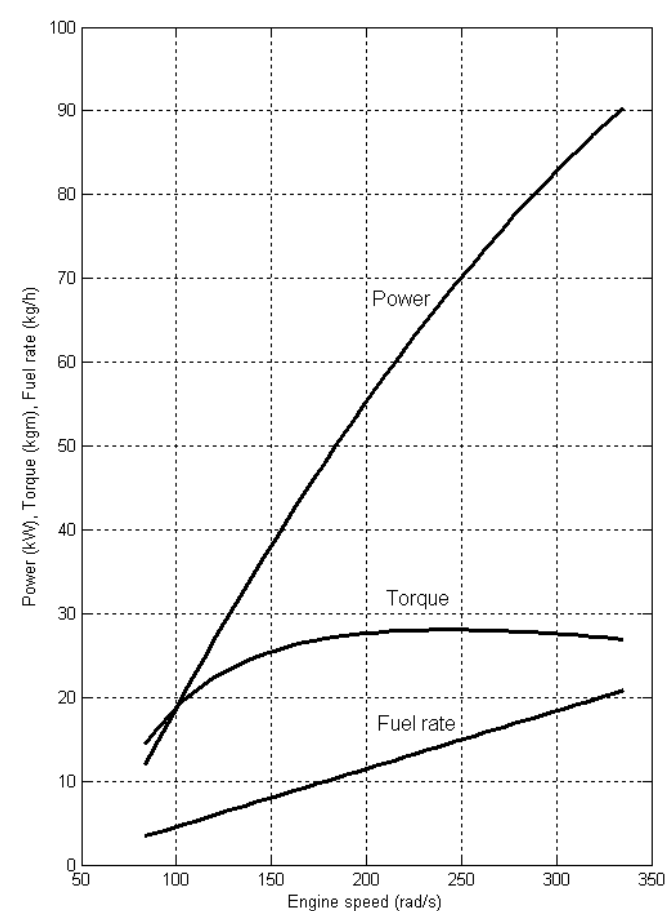

Figure 2: DE characteristics: power $(\mathrm{kW})$, torque $(\mathrm{kg} \bullet \mathrm{m})$ and fuel rate ( $\mathrm{kg} / \mathrm{h})$, all depending on engine speed ( $\mathrm{rad} / \mathrm{s})$

The main simulation test is constant power running on a circle. The task of HMR motion (54) is the circle of radius $125 \mathrm{~m}$, centred at the origin and the equation (55), corresponding to the stabilization of constant power at level $55 \mathrm{~kW}$. Adjustment matrices coefficients (59)-(61) are equal each other and $A=\operatorname{diag}(0.1,0.3,1)$. The initial position of the robot is $Y=\left[\begin{array}{ccc}125 & 0 & \pi / 4\end{array}\right]^{T}$ and the robot does not move. Simulation time is $700 \mathrm{~s}$.

Without loss of generality, we represent the motion resistance as a constant equal torque resistance and changing during the test.

The initial resistance is $640 \mathrm{~N} \cdot \mathrm{m}$. At $225 \mathrm{~s}$ of the simulation, the resistance rises to $740 \mathrm{~N} \cdot \mathrm{m}$ during $5 \mathrm{~s}$. At $380 \mathrm{~s}$ of the simulation, the resistance drop to $540 \mathrm{~N} \cdot \mathrm{m}$ during $20 \mathrm{~s}$. At $500 \mathrm{~s}$ of simulation desired power is changed to $50 \mathrm{~kW}$.

The root-mean-square of position and orientation error should be less than $1 \mathrm{~m}$ at steady state of HMR operation.

The chassis speed and the torque of the traction IM are shown on Fig. 3 and Fig. 4, respectively.

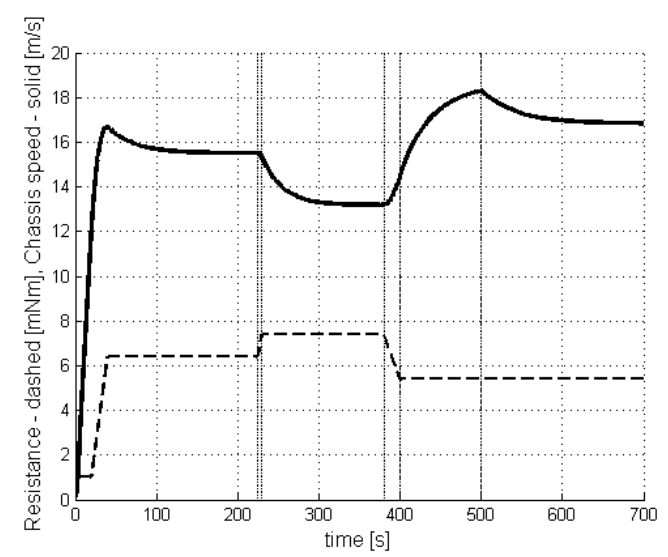

Figure 3: Chassis speed - solid, $(\mathrm{m} / \mathrm{s})$ and motion resistance - dashed $(\mathrm{mN} \bullet \mathrm{m})$ verus time (s) 


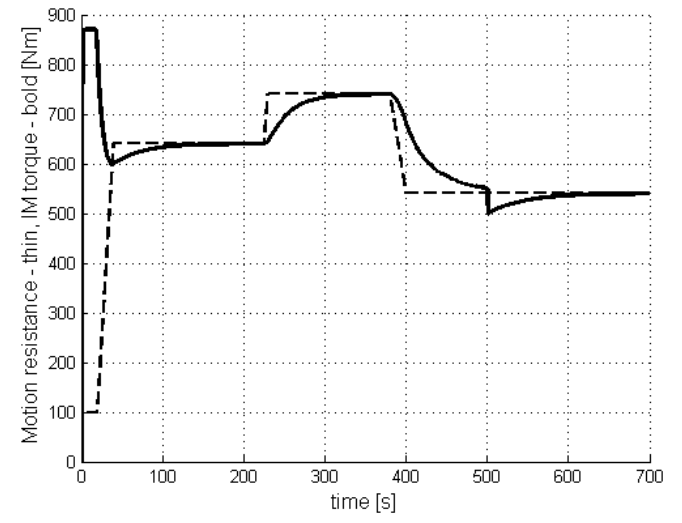

Figure 4: Traction induction motor torque - solid $(\mathrm{N} \bullet \mathrm{m})$ and motion resistance - dashed $(\mathrm{N} \bullet \mathrm{m})$ verus time $(\mathrm{s})$

The figures are shown comparison with the load torque changes. They are both versus the time.

These figures shown the control system (73)-(88) response to unit affects similar that occurs when the vehicle overcoming a hill. Torque change on Fig. 4 at 500 $s$ is result of the desired power change.

The rotor speed and the slip of the traction IM are shown on Fig. 5 and Fig. 6, respectively. They are both versus the time.

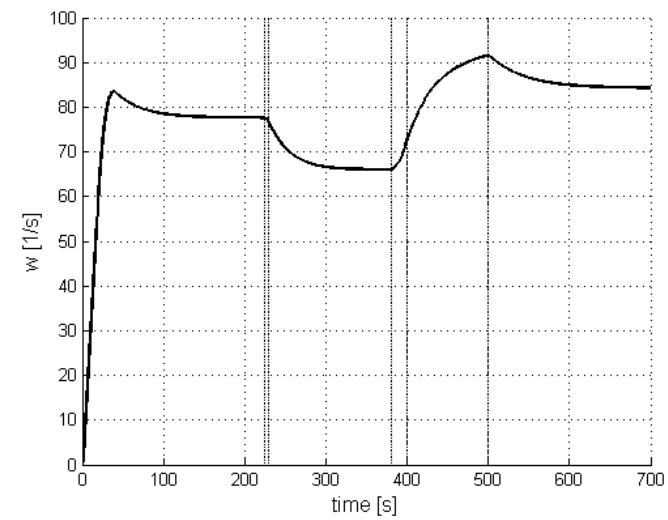

Figure 5: IM rotor rotational speed (1/s) verus time (s)

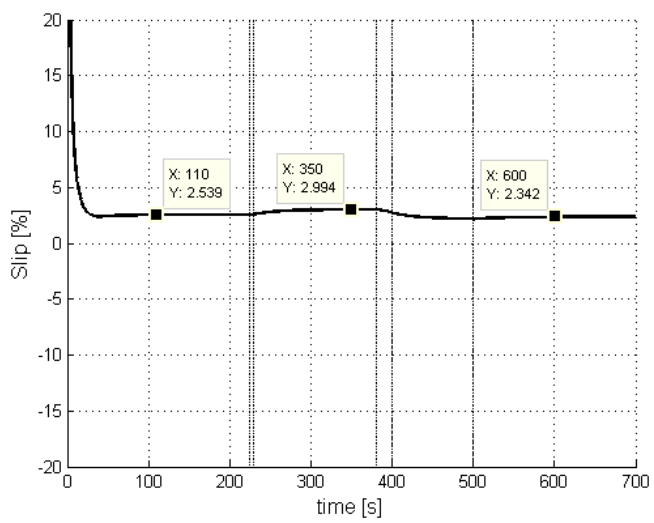

Figure 6: IM slip (\%) verus time (s), steady state slips are exact shown for different areas of the test

The slip is under $3 \%$ that means well efficiency of IM.

The voltage and current of the traction IM are shown on Fig. 7 and Fig. 8, respectively. They are both versus the time. They are shown in $d, q$ reference frame.

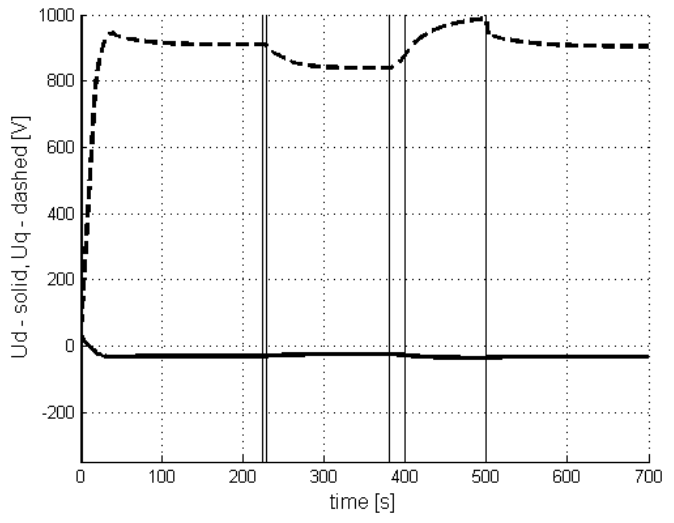

Figure 7: IM voltage (V) in the reference frame $d, q$ verus time (s), $d$-component is solid, $q$-component is dashed

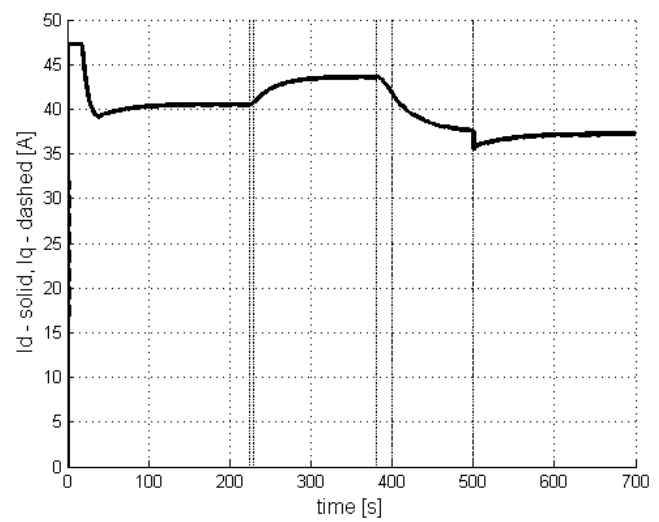

Figure 8: IM current (A) in the reference frame $d, q$ verus time (s), $d$-component is solid, $q$-component is dashed

The rotor flux is optimised during the test. The current components are equal that means minimum amplitude of the stator current. It is effect to minimum copper losses and other losses are neglected for this simulation. This result is nearly to some result, which can be achieved by the known MTA [21] method. If we assume steel losses, the function (43) will not a constant, and $d$ current component with essentialy nonlinear and different from $q$ component.

The rotor flux is on Fig. 9.

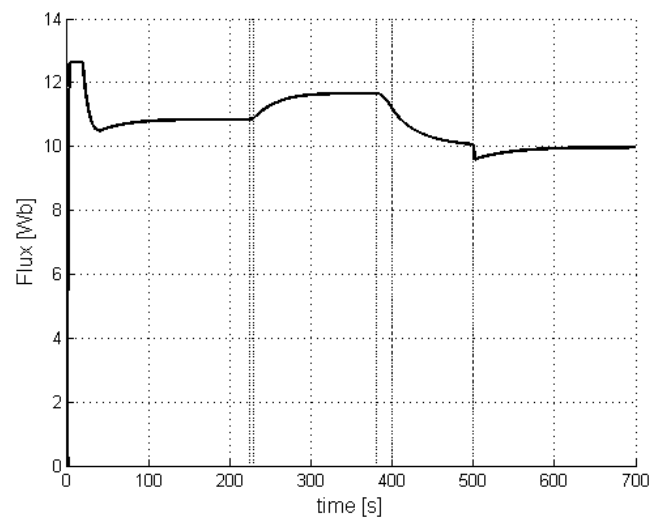

Figure 9: IM rotor flux (Wb) verus time (s)

Power loss of IM is shown on Fig. 10. There is visible a similarity of graphs of current and torque with losses graph. Again, it caused by active losses which are linearly dependent on the current. 


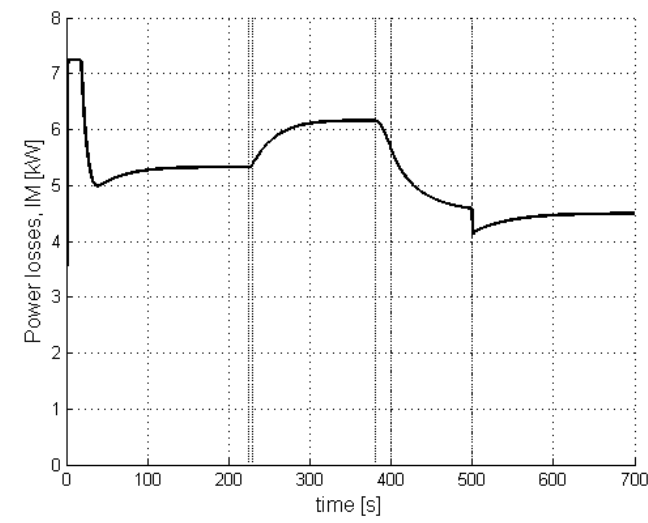

Figure 10: IM power losses $(\mathrm{kW})$ verus time (s)

Consumed power, which is act on the engine shaft is shown on Fig. 11. This power is near to the desired power of the test. The maximum deviation from the desired power is less than $3 \mathrm{rad} / \mathrm{s}$ of the engine shaft speed. This is due to the high inertia of the chassis.

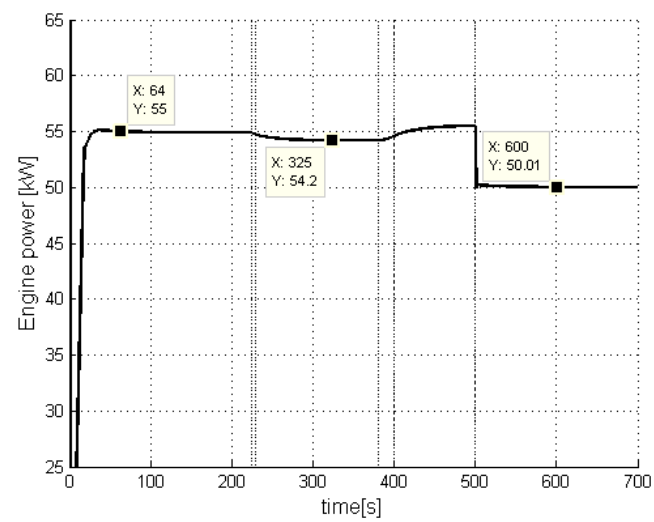

Figure 11: Engine power ( $\mathrm{kW}$ ) verus time (s), deviations are exact shown for different areas of the test

HMR trajectory is shown on Fig. 12 and correspond to desired circle with radius of $125 \mathrm{~m}$.

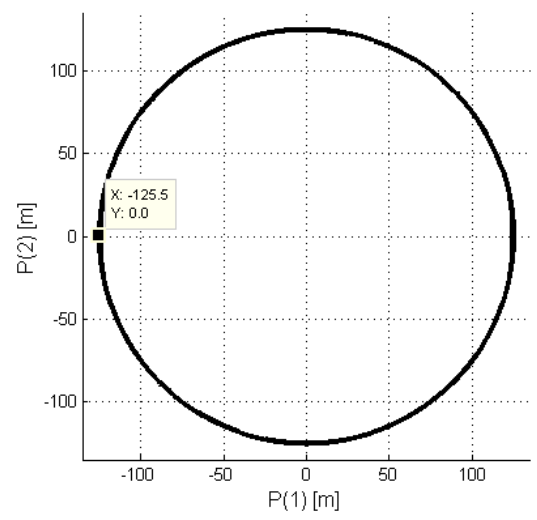

Figure 11: HMR trajecotory, deviation is exact shown

The root-mean-square error is 0.86 at steady state, which correspond to the requirements.

\section{CONCLUSION}

Introduced in this paper solutions extend the Position-trajectory control method for a class of systems, which motion is with a constant power.
The paper introduces solutions to synthesis of nonlinear control algorithms for heave mobile robot with most popular electric transmission.

The solutions are able to optimize energy consumption in the trajectory planning phase of the robot control strategy. This is possible when by taking into account motion resistance changes (parameters of a rough terrain) and as a consequence the necessity of optimization the rotor flux. The introduced solutions are universal about power characteristics of electric machines and to their working mode. This allows the system to control the electric machines with non-hyperbolic characteristics as well as above rated speed without changing the structure of the control system.

The solutions allow to improving the functionality of mobile robots, minimizing fuel consumption without deterioration the quality characteristics, such as accuracy and speed.

\section{ACKNOWLEDGEMENTS}

This work was supported by The Russian Federation President grant HS-1557.2012.10, YD-10982013.10, grant of RFBR 13-08-00315 and The Russian scientific foundation grant 14-19-01533.

\section{REFERENCES}

[1] A.A. Kuleshov, "Powerful vehicles and excavators mining complexes“, Nedra, Moscow (Russia), (1980)

[2] J.B. Heywood, "Internal Combustion Engine Fundamentals”, McGraw-Hill, (USA), (1988).

[3] M. Jankovic, I. Kolmanovsky, “Constructive Lyapunov Control Designfor Turbocharged Diesel Engines," IEEE-transactions on Control Systems Technology, Vol. 8, no. 2, pp. 288-299, (2000)

[4] V. Kh. Pshikhopov, M.Yu. Medvedev, “Control of moving objects in structured and unstructured environment”, Nauka, Moscow (Russia), (2011)

[5] J.Y. Wong, “Theory of Ground Vehicles”, Wiley, (USA), (2008)

[6] C.C. Ward, K. Iagnemma, “A Dynamic-Model-Based Wheel Slip Detector for Mobile Robots on Outdoor Terrain,” Robotics, IEEE Transactions on, Vol.24, no.4, pp.821-831, (2008)

[7] V.V. Pankratov, "Vector Control of Induction Motors", NGTU, Novosibirsk (Russia), (1999)

[8] A.A. Usoltzev, "Frequency Control of Induction Motors”, ITMO, (Russia), (2006)

[9] A.I. Voldek, “Electric Machienes”, Energiya, (USSR) (1978)

[10] A.B. Vinogradov, "Vector Control of AC Motors", IGEU, Ivanovo (Russia), (2008)

[11] I.S. Efremov, "Theory and calculation of electric traction drive”, Vysshaja shkola, Moscow (Russia), (1984)

[12] V.I. Klyuchev, “Theory of Electric Drive”, Energoatomizdat, (USSR), (1998)

[13] M.G. Chilikin, A. S. Sandler, "General Course of Electric Drive”, Energoatomizdat, (USSR), (1981) 
[14] D. Pogosov, "Position-Trajectory Control of Advanced Tracked Robots with Diesel-Electric Powertrain,” Robot Intelligence Technology and Appl. 2, Vol. 274, pp. 393-403, (2014)

[15] D. Yanakiev, I. Kanellakopoulos, “Engine and Transmission Modeling for Heavy-Duty Vehicles”, PATH, (USA) (1995)

[16] Jae Ho Chang, Byung-Kook Kim, “Minimum-time minimum-loss speed control of induction motors under field-oriented control,” Industrial Electronics, IEEE Transactions on, Vol.44, no.6, pp. 809-815, (1997)

[17] Adnene Ben Ali, Riadh Abdelati, M. Faouzi Mimouni, R.Dhifaoui, "Maximum-Torque and MaximumEfficiency Rotor Flux Selection of an Induction Motor in Transient Regime,” International Journal of Sciences and Techniques of Automatic control \& computer engineering; IJ-STA, Vol. 4, no. 2, pp. 1370-1387, (2010)

[18] S. Lim, K. Nam, "Loss-minimising control scheme for induction motors,” Electric Power Applications, IEE Proceedings, Vol.151, no.4, pp.385-397, (2004)

[19] O. Haala, B. Wagner, M. Hofmann, M. Marz, "Optimal current control of externally excited synchronous machines in automotive traction drive applications, “ World Academy of Science, Engineering and Technology, International Science Index 81, International Journal of Electrical, Electronic Science and Engineering, Vol. 7(9), pp. 1-7. (2013)

[20] J. Ramirez, C. Canudas de Wit, “Optimal TorqueFlux Control of Induction Motors: Experimental Evaluation,” Proc. of the Electrimac congress, Vol. 2, pp. 763-768
[21] O. Wasynczuk, S.D. Sudhoff, K.A. Corzine, J.L. Tichenor, P.C. Krause, I.G. Hansen, and L.M. Taylor, “A Maximum Torque Per Ampere Control Strategy for Induction Motor Drives,” IEEE Transactions on Energy Conversion, Vol. 13, no. 2, pp. 163-169, (1998)

[22] D. Rodkin, V. Chenchevoy, V. Ogar, "Determination of losses in steel deep induction motor with its saturated," Kremenchuk Mykhailo Ostrohradskyi National University, Journal Elekromechanichni i energosberegauchi sistemi. Vol. 2 (22) pp. 75-85, (2013)

[23] V.Kh. Pshikhopov, V.A. Krukhmalev, M. Yu. Medvedev, R.V. Fedorenko, S.A. Kopylov, A.Yu. Budko, V.M. Chufistov, “Adaptive Control System Design for Robotic Aircrafts," Robotics Symposium and Competition (LARS/LARC), Latin American, pp. 67-70, 21-27 Oct. (2013)

[24] V. Pshikhopov, N. Sergeev, M. Medvedev and A. Kulchenko, “The Design of Helicopter Autopilot,” SAE Technical Paper 2012-01-2098, (2012)

[25] V. Pshikhopov, M. Medvedev, V. Kostjukov, R. Fedorenko et al., "Airship Autopilot Design," SAE Technical Paper 2011-01-2736, (2011)

[26] V.Kh. Pshikhopov, M.Y. Medvedev, "Robust control of nonlinear dynamic systems,” ANDESCON, 2010 IEEE, pp.1,7, 15-17 (2010)

[27] D. Pogosov, “Transformation of High-order Splinefunctions to implicit form,” SWorld conference proceedings 3, Vol. 4, pp. 84-87, (2012) 